Jean-Louis

Vanoverschelde

Christophe Depré

William Wijns

Anne Bol

Robert Dion

Bernhard Gerber

Marcel Borgers

Jacques Melin

\title{
Physiopathologie de I'hibernation myocardique chronique: apports de la tomographie par émission de positrons
}

Chez certains malades coronariens, le dysfonctionnement régional du ventricule gauche est réversible après restauration du flux sanguin par pontage artériel chirurgical. Cette condition a été appelée hibernation myocardique. Deux groupes de malades présentant une dysfonction ventriculaire segmentaire ont été étudiés, ayant ou non des antédents d'infarctus. Chez les malades n'ayant pas eu d'infarctus, le débit sanguin régional, le métabolisme glucidique et la consommation d'oxygène étaient normaux dans les régions dysfonctionnelles. En revanche, la réserve de débit collatéral était très diminuée, ce qui suggère que les épisodes répétés d'ischémie engendrent un état de sidération qui, devenant permanent, conduit à l'hibernation. Les différences ultrastructurales entre dysfonction par hibernation et dysfonction post-infarctus reposent, respectivement, sur l'accumulation de glycogène, la diminution des myofibrilles, des aspects de différenciation cellulaire dans l'hibernation, la fibrose après infarctus. La vitesse de récupération fonctionnelle après revascularisation est étroitement liée à la sévérité des anomalies ultrastructurales.

\footnotetext{
ADRESSE

J.L. Vanoverschelde: docteur en médecine, agrégé ; C. Depré : docteur en médecine; W. Wijns : docteur en médecine, professeur agrégé de médecine ; A. Bol: docteur en physique ; R. Dion: docteur en médecine, professeur de médecine; B. Gerber: docteur en médecine; M. Borgers : docteur en sciences, professeur agrégé ; J. Melin: docteur en médecine, professeur agrégé de médeci$n e$. Service de cardiologie et unité de tomographie par émission de positrons, université catholique de Louvain, cliniques universitaires St-Luc, avenue Hippocrate, 10-2881, B-1200 Bruxelles, Belgique.

ar son activité mécanique incessante et ses besoins énergétiques considérables, le cour puise l'essentiel de son énergie dans le métabolisme aérobie. Dans ces conditions, toute réduction des apports sanguins (ou ischémie) par l'athérosclérose des artères coronaires s'accompagne d'une réduction immédiate et proportionnelle de la force de contraction régionale. Lorsqu'une telle ischémie se prolonge au-delà de quelques minutes, les cellules myocardiques subissent des lésions irréversibles, dont l'étendue et la sévérité sont proportionnelles à la durée et à l'importance de la réduction du flux sanguin myocardique [1]. On parle alors de nécrose ou d'infarctus du myocarde. Jusqu'à il y a peu, la découverte d'une dysfonction myocardique régionale en l'absence d'ischémie active chez un malade coronarien était considérée comme témoin d'une nécrose myocardique 


\section{RÉFÉRENCES}

1. Jennings RB, Murry CE, Steenbergen C Reimer KA. Development of cell injury in sustained acute ischemia. Circulation 1990 . 82 (suppl. II) : 2-12.

2. Kloner RA, Przyklenk K, Patal B. Altered myocardial states. The stunned and hibernating myocardium. Am J Med 1989; 86 (suppl. 1A) : 14-22.

3. Braunwald E, Rutherford JD. Reversible ischemic left ventricular dysfunction: evidence for «hibernating myocardium ". $J A m$ Coll Cardiol 1986; 8: 1467-70.

4. Heyndrickx GR, Millard RW, McRitchie RJ, Maroko PR, Vatner SF. Regional myocardial functional and electrophysiological alterations after brief coronary occlusions in conscious dogs. J Clin Invest 1975; 56 : 97885

5. Heyndrickx GR, Wijns W, Vogelaers D Degrieck Y, Bol A, Vandeplassche G, Melin JA. Recovery of regional contractile function and oxidative metabolism in stunned myocardium induced by l-hour circumflex coronary artery stenosis in chronically ins trumented dogs. Circ Res 1993; 72: 901-13.

6. Bolli R. Myocardial «stunning" in man Circulation 1992; 86: 1671-91.

7. Rahimtoola SH. The hibernating myocardium. Am Heart J 1989; 117: 211-21

8. Vanoverschelde JLJ, Wijns W, Depré C, et al. Mechanisms of chronic regional post-
ischemic dysfunction in humans: New insights from the study of non-infarcted collateral dependent myocardium. Circulation $1993 ; 87: 1513-23$

9. Depré C, Vanoverschelde JL, Melin JA Borgers M, Bol A, Ausma J, Dion R, Wijn W. Structural and metabolic correlates of the reversibility of chronic left ventricula ischemic dysfunction in humans. $A m / P h y$ arrivée au stade cicatriciel. L'introduction des techniques de revascularisation myocardique, soit par pontage chirurgical, soit par angioplastie percutanée, a cependant modifié cette vision des choses. Il n'est, en effet, pas rare d'observer, après revascularisation, une amélioration, voire une normalisation, de la cinétique seg. mentaire au sein de territoires qui, du fait de leur dysfonction, auraient été considérés comme nécrotiques ou cicatriciels auparavant. Ces observations ont conduit à l'émergence de deux nouveaux concepts physiopathologiques: la sidération et l'hibernation myocardiques $[2,3]$. La sidération myocardique est une forme de dysfonction myocardique réversible qui fait suite à un épisode d'ischémie transitoire, de brève durée, typiquement quinze minutes d'occlusion coronaire chez le chien [4]. Dans ces conditions, et bien que l'ischémie n'ait pas été de durée suffisante pour entraîner des dégâts cellulaires irréversibles, la reperfusion ne permet pas de rétablir d'emblée une fonction contractile normale. Celle-ci peut mettre jusqu'à deux semaines avant de se normaliser [5]. Cette situation, décrite initialement chez le chien au cours d'expériences d'occlusion coronaire transitoire, peut également se rencontrer chez le malade coronarien, par exemple lors d'épisodes d'angor instable ou après thrombolyse pour infarctus du myocarde [6].

Le concept d'hibernation myocardique fut, quant à lui, introduit par Rahimtoola pour décrire un état de dysfonction ventriculaire gauche régionale chronique, associé à la maladie coronarienne et réversible après pontage chirurgical [7]. Les mécanismes physiopathologiques qui sous-tendent l'hibernation myocardique sont encore mal connus, sans doute parce qu'il n'a jamais été possible jusqu'à présent de reproduire expérimentalement une condition similaire chez l'animal. L'hypothèse initialement avancée par Rahimtoola supposait que la dysfonction segmentaire observée en l'absence d'infarctus était secondaire à une réduction chronique de la perfusion myocardique au sein de ce territoire. Cette hypothèse présupposait l'établissement d'un nouvel état d'équilibre entre les besoins myocardiques en oxygène (travail contractile) et les apports sanguins artériels (réduits par la maladie coronaire), par le biais d'une réduction spontanée du travail cardiaque.

En l'absence d'un modèle animal adéquat pour l'hibernation myocardique, nous avons entrepris d'étudier plus avant les mécanismes physiopathologiques qui sous-tendent l'hibernation myocardique, chez le malade coronarien $[8,9]$. Nous nous sommes adressés à deux populations de malades chez lesquels la maladie coronaire s'était compliquée de dysfonction régionale. Dans un premier temps [8], nous nous sommes intéressés à des malades qui n'avaient jamais présenté d'infarctus du myocarde auparavant et chez lesquels existait pourtant une occlusion complète de l'artère interventriculaire antérieure, l'une des trois principales artères coronaires. Chez ces malades, la paroi antérieure du ventricule gauche, qui est normalement vascularisée par l'artère interventriculaire antérieure, était devenue dépendante d'une circulation collatérale de vicariance en provenance des deux autres artères. Malgré l'absence d'antécédents d'infarctus (à l'interrogatoire comme à l'électrocardiogramme), plusieurs de ces malades présentaient une dysfonction sévère de leur paroi antérieure à la ventriculographie de contraste. Ces malades pouvaient donc être considérés comme porteurs de myocarde hibernant. Malheureusement, ce type de malades est relativement rare et ne représente qu'une faible proportion de ceux chez lesquels on découvre une dysfonction ventriculaire segmentaire. La plupart de ceux qui nous consultent ont bien souvent déjà présenté un ou plusieurs épisodes d'infarctus du myocarde. Dans un second temps [9], nous nous sommes donc intéressés à une population de malades plus représentative du malade coronarien "quotidien", chez lequel on se pose la question de savoir si la dysfonction segmentaire est le fait d'une nécrose myocardique irréversible ou d'un état d'hibernation myocardique susceptible de s'améliorer après revascularisation.

Chez tous ces malades, nous avons étudié la perfusion myocardique, la consommation myocardique en oxygène et l'utilisation myocardique du 
glucose, au sein des zones normales et des zones dysfonctionnelles, grâce à la tomographie par émission de positrons (TEP), une technique d'imagerie nucléaire permettant l'étude et la quantification absolue de phénomènes physiologiques et métaboliques régionaux [10-13]. Chez certains malades, nous avons également pu évaluer la réserve de vasodilatation coronaire au sein du territoire dysfonctionnel, en mesurant le flux sanguin régional avant et après l'administration intraveineuse d'un vasodilatateur artériolaire, le dipyridamole. En outre, chez un nombre limité de malades, un prélèvement biopsique a pu être obtenu au sein de la zone dysfonctionnelle, au moment de l'intervention de pontage chirurgical. Enfin, chez tous ces malades, l'amplitude et la cinétique de la récupération fonctionnelle après revascularisation ont pu être étudiées, apportant ainsi la confirmation a posteriori qu'il s'agissait bien de myocarde hibernant.

\section{Dysfonction segmentaire chronique du myocarde dépendant de collatérales en l'absence d'infarctus}

Nous avons étudié 26 malades ( 19 hommes et 7 femmes, d'un âge moyen de $60 \pm 9$ ans), tous porteurs d'une occlusion complète de l'artère interventriculaire antérieure [8]. Aucun de ces malades n'avait présenté, sur bases de critères cliniques et électrocardiographiques, d'inf arctus du myocarde auparavant. La TEP a été utilisée pour étudier, en valeurs absolues, par unité de masse myocardique, la perfusion, la consommation d'oxygène et le captage de glucose au sein des segments normaux et dysfonctionnels. Nous avons utilisé l'ammoniaque $\mathrm{N}-13$ comme traceur de perfusion, l'acétate C-1l comme traceur du cycle de Krebs et le fluorodésoxyglucose F-18 comme traceur des premières étapes de la glycolyse (captage et phosphorylation par l'hexokinase). Chez onze malades, la

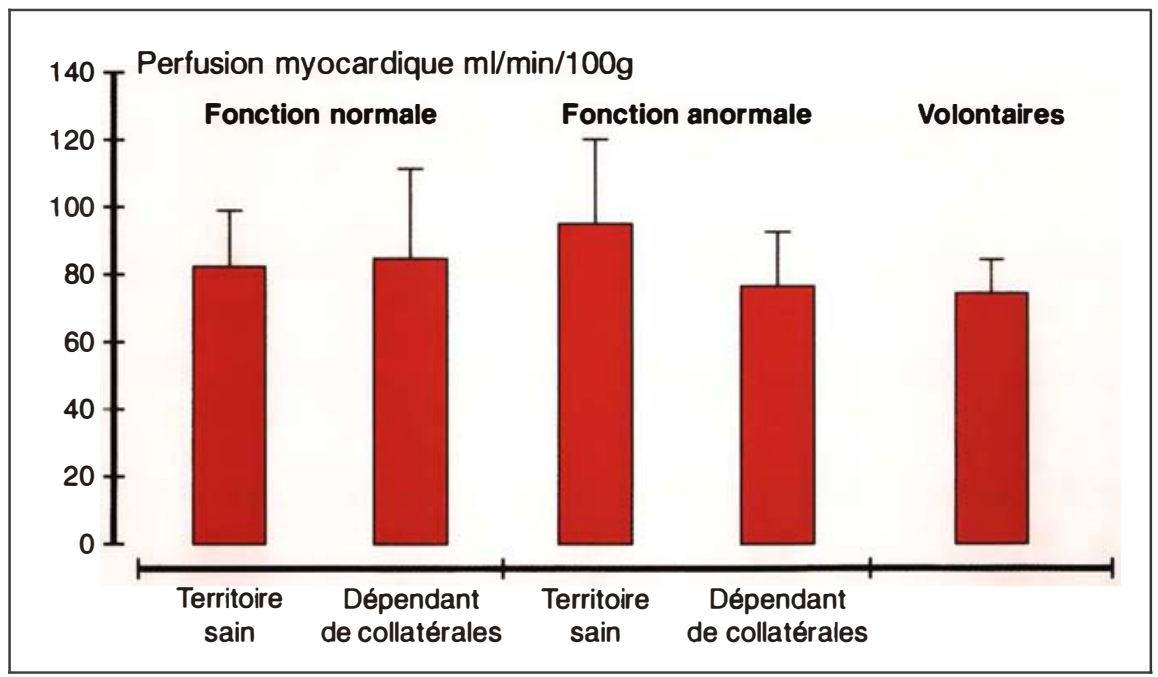

Figure 1. Perfusion myocardique au repos, exprimée en valeurs absolues $(\mathrm{ml} / \mathrm{min} / 100 \mathrm{~g}) \pm 1$ déviation standard (SD), mesurée à l'aide d'ammoniaque $\mathrm{N}-13$, dans les territoires sains ou dépendant de collatérales chez des malades coronariens ayant une artère coronaire occluse et présentant ou non une fonction segmentaire normale dans le territoire dépendant de collatérales. Ces résultats sont comparés à ceux obtenus dans un groupe de volontaires normaux. La perfusion des zones dysfonctionnelles dépendant de collatérales n'est pas différente de celle des volontaires normaux ou des malades dont la fonction est normale. réserve de perfusion tissulaire a été évaluée après administration intraveineuse de $0,84 \mathrm{mg} / \mathrm{kg}$ de dipyridamole. Chez tous ces malades, la fonction ventriculaire gauche globale et régionale a été évaluée par ventriculographie de contraste, au moment de l'étude TEP, de même que six mois après revascularisation.

Sur base de la cinétique de la paroi ventriculaire antérieure à l'état basal, les malades ont été classés en deux groupes: un premier groupe, de 9 malades (8 hommes et 1 femme, d'un âge moyen de $60 \pm 7$ ans), chez lesquels la fonction ventriculaire, tant globale que régionale, était normale, malgré l'occlusion artérielle, et un second groupe, de 17 malades ( 11 hommes et 6 femmes, d'un âge moyen de $61 \pm 9$ ans), chez lesquels il existait une dysfonction sévère (akinésie complète) dans le territoire de l'artère occluse.

\section{Flux et métabolisme à l'état basal}

La perfusion myocardique, la consommation d'oxygène et le captage de glucose ont été étudiés chez tous les malades à l'état basal, dans la zone dépendant de collatérales et dans une zone de référence normale. Chez les malades ne présentant pas de dysfonction régionale, la perfusion myocardique était similaire au sein des régions normales ou dépendant de collatérales, respectivement $83 \pm 18$ et $85 \pm 14 \mathrm{ml} / \mathrm{min} / 100 \mathrm{~g}$. Ces valeurs ne sont pas différentes de celles obtenues dans un groupe de volontaires normaux, exempts de maladie coronarienne $(75 \pm 10 \mathrm{ml} / \mathrm{min} / 100 \mathrm{~g})$. En revanche, chez les malades porteurs d'une dysfonction segmentaire du territoire dépendant de collatérales, la perfusion myocardique était plus basse dans le territoire dépendant de collatérales $(77 \pm 25 \mathrm{ml} / \mathrm{min} / 100 \mathrm{~g})$ que dans la zone de référence normale $(96 \pm 26 \mathrm{ml} / \mathrm{min} / 100 \mathrm{~g}, \mathrm{p}<0,00 \mathrm{l})$ Néanmoins, les valeurs obtenues au sein des zones dysfonctionnelles dépendant de collatérales n'étaient pas différentes de celles observées au sein des mêmes territoires chez les malades sans dysfonction régionale ou chez des volontaires normaux (figure 1). Dans cette population sélectionnée, l'hibernation myocardique ne s'accompagne donc pas d'une réduction de la perfusion myo- 


\section{RÉFÉRENCES}

10. Bol A, Melin JA, Vanoverschelde JL, Baudhuin T, Vogelaers D, De Pauw $M$, Michel C, Luxen A, Labar D, Cogneau M, Robert A, Heyndrickx GR, Wijns W. Direct comparison of $\mathrm{N}-13$ ammonia and $\mathrm{O}-15$ water estimates of perfusion with quantitation of regional myocardial blood flow by microspheres. Circulation 1993; 87: 512-25.

11. Wijns W, Heyndrickx GR, Vogelaers D, Vanoverschelde JL, Bol A, Essamri B, Grandin $C$, Melin JA. Metabolic imaging in postischemic canine myocardium. In : Zaret BL Beller GA, eds. Nuclear cardiology: state of the art and future directions. St Louis: Mosby, 1993: 284-93.

12. Vanoverschelde JL, Wijns W, Kolanowski I, Bol A, DeCoster P, Michel C, Cogneau M, Heyndrickx GR, Essamri B, Melin JA. Competition between palmitate and ketone bodies for the human heart: a study with positron emission tomography. Am J Physiol 1993 ; 264 : H701-7.

13. Vanoverschelde JL, Melin J, Bol A, Vanbutsele R, Cogneau M, Labar D, Robert A, Michel C, Wijns W. Regional oxidative metabolism in patients after recovery from reperfused anterior myocardial infarction: relation to regional blood flow and glucose metabolism. Circulation 1992; 85 : 9-21.

14.Borgers M, Thoné F, Wouters L, Ausma J, Shivalkar B, Flameng W. Structural correlates of regional myocardial dysfunction in patients with critical coronary artery stenosis: chronic hibernation? Cardiovasc Pathol $1993 ; 2: 237-45$.

15. Ausma J, Schaart G, Thoné F, Shivalkar B, Flameng W, Depré C, Vanoverschelde JL, Ramaekers F, Borgers M. Chronic ischemic viable myocardium: aspects of dedifferentiation. Cardiovasc Pathol 1995; 4 : 29-37.

16. Guerre-Millo M. Les transporteurs d'hexoses. médecine/sciences 1995; 11: 1111 9.

17. Vanoverschelde JL, Melin JA, Depré C, Borgers M, Dion R, Wijns W. Time-course of functional recovery of hibernating myocardium after coronary revascularization. cardique, telle qu'initialement proposée par Rahimtoola [7]. De même, l'étude de la consommation d'oxygène régionale n'a pas permis de mettre en évidence de différences notables entre les régions dépendant de collatérales, qu'elles soient dysfonctionnelles ou non. Cela confirme que, contrairement à l'hypothèse initiale, la consommation d'oxygène de ces segments n'est pas réduite. Ainsi, la consommation d'oxygène régionale était-elle similaire au sein des territoires dépendant de collatérales et présentant $(85 \pm 18 \mathrm{~J} / \mathrm{min} / 100 \mathrm{~g})$ ou non $(95 \pm 25 \mathrm{~J} / \mathrm{min} / 100 \mathrm{~g})$ une dysfonction régionale. En outre, chez un même malade, le captage de glucose exogène était semblable au sein des zones normales ou dépendant de collatérales d'un même malade. Il existait cependant des différences appréciables entre les deux groupes de malades, ceux présentant une dysfonction segmentaire ayant un captage de glucose significativement plus élevé que ceux dont la fonction était normale. Il faut cependant noter que nous avons étudié le captage myocardique de glucose après surcharge intraveineuse en glucose, lorsqu'il est essentiellement dépendant de l'action de l'insuline. Cela explique sans doute que nos résultats diffèrent de ceux obtenus lorsque le captage de glucose est étudié à jeun. Dans ces conditions, le captage de glucose est indépendant de l'action de l'insuline et les valeurs mesurées sont considérablement plus faibles. Lorsque des malades porteurs de myocarde hibernant sont étudiés à jeun, on peut généralement observer un captage accru de glucose dans les zones dysfonctionnelles par rapport aux zones normales.

\section{Réserve de perfusion myocardique}

Le fait d'observer que le niveau de perfusion basale est semblable au sein des territoires dépendant de collatérales, qu'ils présentent ou non une dysfonction segmentaire, remet en question l'hypothèse selon laquelle l'hibernation myocardique serait secondaire à une réduction chronique de la perfusion tissulaire. Si tel n'est pas le cas, une autre hypothèse peut être proposée sous forme d'alternative: soit l'hibernation myocardique est l'expression de méca- nismes déjà connus, comme par exemple la sidération myocardique, soit elle est le fait d'un mécanisme encore inconnu. De façon pragmatique, nous avons testé l'hypothèse selon laquelle l'hibernation pouvait être secondaire à des épisodes répétés de sidération myocardique, dont la fréquence serait telle que le myocarde n'a pas le temps de récupérer dans l'intervalle libre. Pour étayer cette hypothèse, nous avons évalué la réserve de perfusion myocardique au sein des territoires dépendant de collatérales, dans l'idée que la survenue d'épisodes fréquents d'ischémie myocardique devait trouver son origine dans l'adaptation insuffisante du débit coronaire au cours d'un stress et donc d'une réduction de la réserve de vasodilatation coronaire. La réserve de perfusion a donc été étudiée grâce à la TEP, couplée à la mesure des débits par l'ammoniaque $\mathrm{N}-13$, au cours de l'administration intraveineuse de dipyridamole.

De façon surprenante, malgré l'occlusion complète de l'artère nourricière, la réserve de perfusion coronaire s'avéra semblable au sein du territoire dépendant de collatérales et du territoire normal des malades dont la fonction segmentaire était normale (figure 2). En moyenne, sous dipyridamole, le flux maximal s'élevait à $274 \pm 44 \mathrm{ml} /$ $\min / 100 \mathrm{~g}(3,2 \pm 0,8$ fois le flux basal $)$ dans la zone normale et à $238 \pm$ $54 \mathrm{ml} / \mathrm{min} / \mathrm{l} 00 \mathrm{~g}(3,0 \pm 0,5$ fois le flux basal) dans la zone dépendant de collatérales. En revanche, chez les malades présentant une dysfonction segmentaire dans le territoire dépendant de collatérales, le flux maximal n'atteignait que $112 \pm 44 \mathrm{ml} / \mathrm{min} / 100 \mathrm{~g}$ ( $1,4 \pm 0,8$ fois le flux basal). De plus, alors qu'il n'existait aucune relation entre le niveau de perfusion basale et la fonction segmentaire, il existait une relation significative $(r=-0,85$, $p<0,001$ ) entre la réserve de perfusion coronaire et la fonction contractile segmentaire (figure 3). Cela confirme donc que, chez les malades porteurs de myocarde hibernant, la dysfonction segmentaire n'est pas le fait d'une réduction chronique de la perfusion basale mais serait plutôt liée à une réduction de la réserve perfusionnelle; celle-ci pourrait entraîner non seulement davantage, mais peutêtre aussi de plus longs épi- 


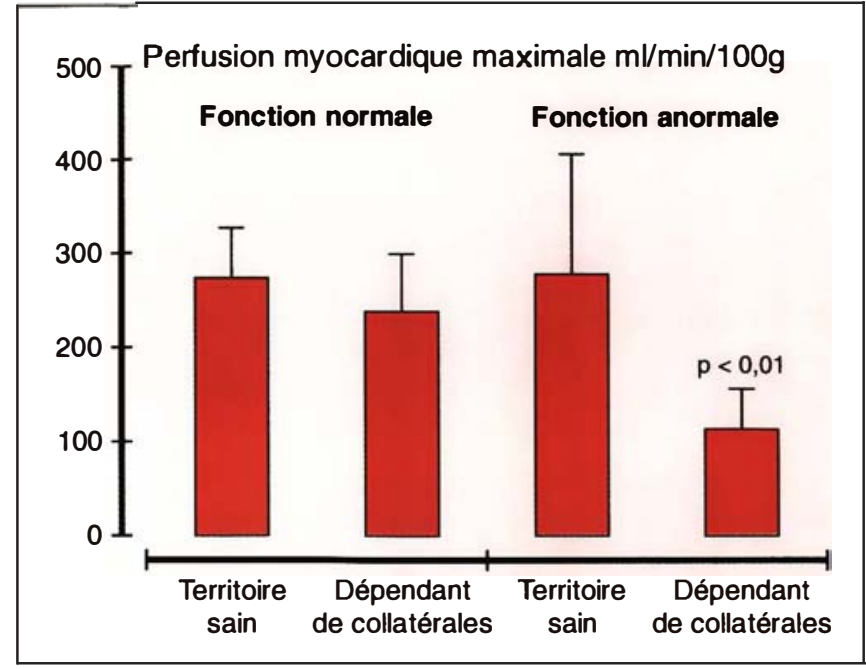

Figure 2. Perfusion myocardique maximale mesurée après administration intraveineuse de dipyridamole au sein des territoires sains ou dépendant de collatérales de malades coronariens ayant une artère coronaire occluse et présentant ou non une fonction segmentaire normale dans le territoire dépendant de collatérales. La perfusion maximale est nettement diminuée dans les régions dysfonctionnelles dépendant de collatérales.

sodes ischémiques et, par conséquent, de sidération myocardique post-ischémique.

\section{Corrélations ultrastructurales}

Si, sur la base des observations précédentes, il apparaissait que l'hibernation myocardique chronique est sans doute le fait d'épisodes répétés de sidération myocardique dont le myocarde n'a jamais le temps de se remettre complètement, restait encore à déterminer quel impact ce phénomène pouvait avoir sur l'intégrité tissulaire. Pour répondre à cette question, nous avons obtenu des biopsies myocardiques transmurales prélevées au sein de la zone dysfonctionnelle lors des interventions de pontage aorto-coronaire dont ont bénéficié certains malades. Ces prélèvements biopsiques ont été examinés au microscope optique (après coloration par le bleu de toluidine et le PAS) et au microscope électronique $[8,14,15]$. Certaines biopsies ont également été congelées dans l'isopentane (et étudiées par immunohistochimie) ou dans l'azote liquide (pour en isoler l'ARN messager des différents transporteurs de glucose). Dans aucun des cas analysés, nous n'avons pu observer de zone cicatricielle (qui aurait pu signer la présen-

$\mathrm{m} / \mathrm{s} n^{\circ} 9$, vol. 11, septembre 95 ce d'un infarctus ancien) ni d'augmentation importante de la trame interstitielle. En revanche, il existait de façon constante, au sein des territoires dysfonctionnels dépendant de collatérales, des anomalies structurales au niveau des cardiomyocytes. Ces anomalies comprenaient: (1) une diminution du contenu en matériel contractile, plus marquée dans les régions périnucléaires. Dans les cellules les plus affectées, les quelques sarcomères restants étaient le plus souvent situés à la périphérie cellulaire; (2) une accumulation massive, au centre du cardiomyocyte, d'un matériel amorphe, coloré intensément par le PAS, correspondant à du glycogène; (3) la disparition du réticulum sarcoplasmique et des tubules T; (4) des modifications de la structure des mitochondries qui deviennent plus petites et souvent allongées et perdent leur segmentation habituelle (figure 4). Dans aucun cas, nous n'avons pu observer de changements structuraux suggérant une dégénérescence cellulaire ou des dégâts ischémiques. En moyenne, ces anomalies atteignaient environ $50 \%$ des cardiomyocytes examinés, les $50 \%$ restants présentant un aspect morphologique strictement normal [8]. En immunohistochimie, l'étude de la présence et du mode d'organi- sation de certaines protéines contractiles ou structurales a permis de montrer que les cardiomyocytes "hibernants" évoluaient vers un phénotype fœtal [15]. Ainsi ces cardiomyocytes réexpriment-ils l' $\alpha$-actine musculaire lisse, une isoforme de l'actine dont les cardiomyocytes adultes sont normalement dépourvus, mais que l'on retrouve communément au sein des cardiomyocytes fœtaux ou au cours de la vie embryonnaire. Les cardiomyocytes présentaient aussi d'autres caractéristiques du muscle cardiaque fotal, entre autres l'absence de cardiotine, une protéine structurale de haut poids moléculaire qui s'accumule dans le cœur après la naissance. De même, la distribution intracellulaire de titine était caractéristique de celle rencontrée dans le cœur fœtal et très différente de celle normalement rencontrée dans le cœur adulte

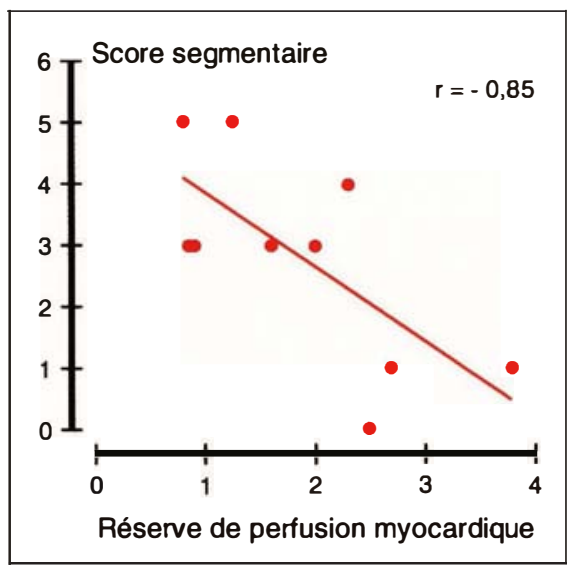

Figure 3. Corrélation entre la réserve de perfusion myocardique mesurée dans les régions dépendantes de collatérales et le score de fonction segmentaire (qui quantifie l'amplitude et l'étendue de la dysfonction). Une relation inverse significative est observée entre ces deux paramètres.

[15]. Enfin nous avons pu montrer par PCR, de manière préliminaire, que le myocarde hibernant réexprimait le transporteur de glucose unidirectionnel GLUT-1, dont le cœur adulte est presque dépourvu et qui constitue le transporteur préféré du cœur fœtal (le cœur adulte exprime plus volontiers le transporteur sen- 

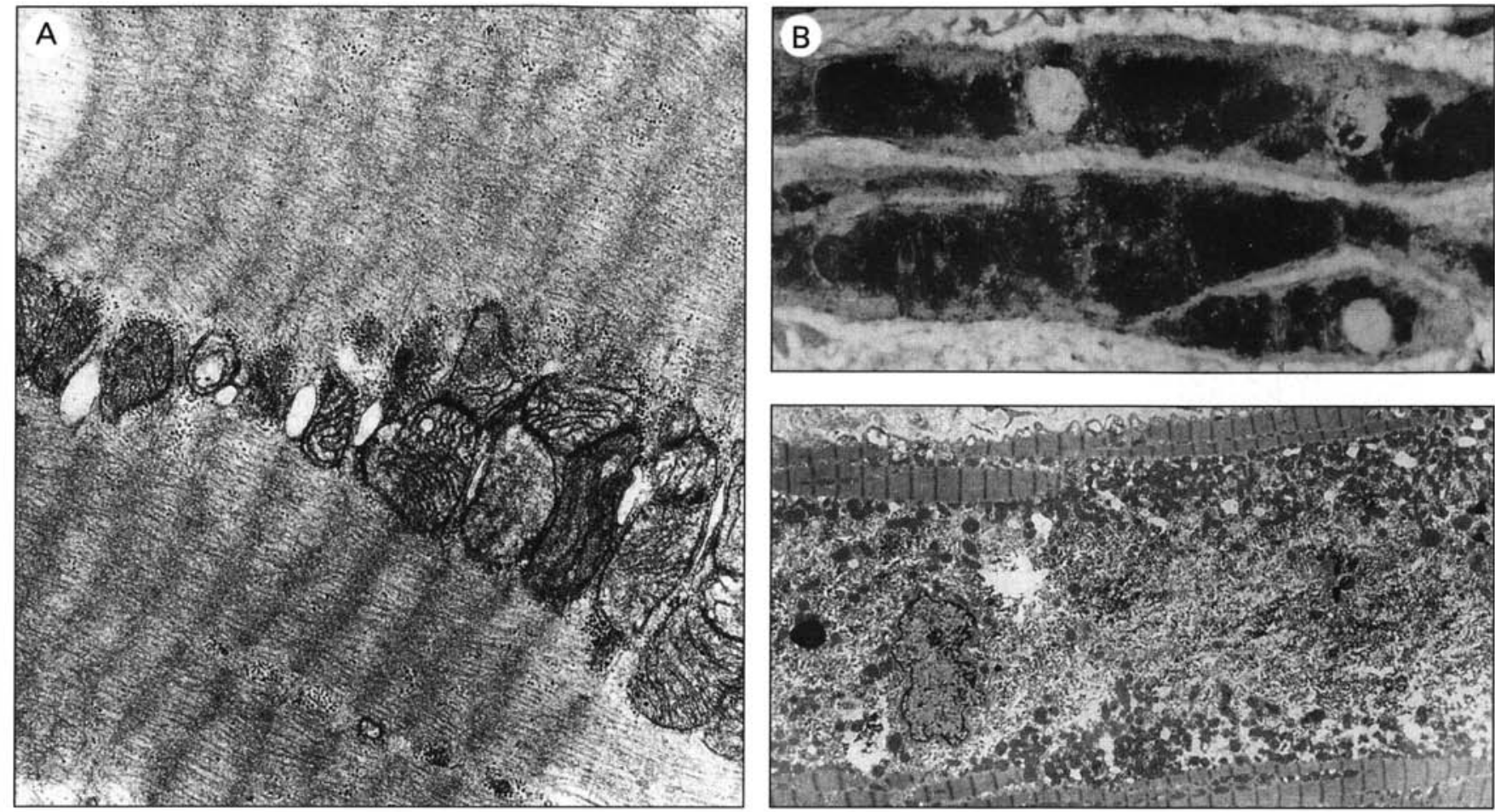

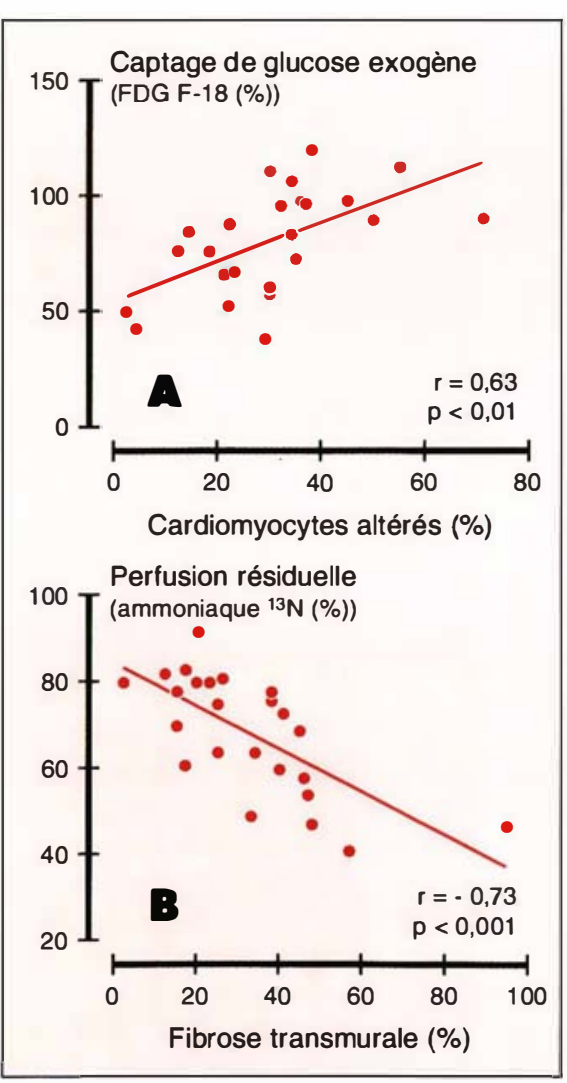

1320

\section{$\Delta$}

Figure 4. Exemples de cardiomyocyte normal (photo A) et altéré (photo $B$ ) visualisés en microscopie optique (photo $B$, partie supérieure) et électronique (photo $A$; photo $B$, partie inférieure). II convient de noter l'importante diminution du contenu en myofilaments de même que l'accumulation de matériel amorphe, granulaire, coloré intensément par le PAS au sein des cardiomyocytes altérés.

Figure 5. Corrélations linéaires entre le nombre de cardiomyocytes altérés et le captage de glucose exogène, d'une part (graphe A) et entre la sévérité de la fibrose et le degré de perfusion résiduelle (graphe $B$ ), d'autre part. FDG F-18: fluorodésoxyglucose F18, marqueur des premières étapes de la glycolyse (captage et phosphorylation). Les altérations des cardiomyocytes sont à type de diminution du matériel contractile, d'accumulation de glycogène, de disparition du réticulum sarcoplasmique et des tubules $T$, d'anomalies morphologiques des mitochondries. $n=24$.

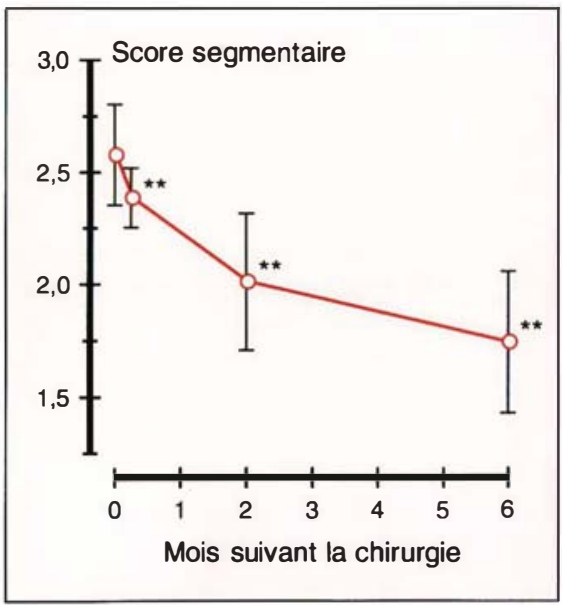

Figure 6. Évolution de la fonction contractile segmentaire, évaluée par le score segmentaire, au cours des six premiers mois suivant l'acte de revascularisation, chez les malades à la dysfonction réversible $(n=18)$. Le score segmentaire est évalué par le score de mouvement pariétal segmentaire à l'échocardiographie. La récupération de fonction se fait de manière progressive au cours des six premiers mois. 
sible à l'insuline GLUT-4). La réexpression de GLUT-1 au sein du myocarde hibernant constitue sans doute la base moléculaire du captage accru de glucose, indépendant de l'insuline, qui caractérise l'hibernation et sans doute aussi celle de l'accumulation de glycogène [16]. Des travaux récents portant sur des souris transgéniques exprimant en excès GLUT1 dans le muscle strié squelettique ont montré que cette surexpression s'accompagnait d'une accumulation de glycogène dans les muscles concernés.

\section{Corrélations métaboliques, perfusionnelles et structurales de la dysfonction segmentaire réversible après revascularisation chez le malade coronarien}

Les résultats précédents ont été obtenus dans une population sélectionnée de malades coronariens sans infarctus. Ils nous ont permis de formuler une hypothèse nouvelle et originale selon laquelle l'hibernation myocardique serait le résultat de l'adaptation du myocarde à des épisodes fréquents et répétés d'ischémie myocardique suivis de sidérations. Cette adaptation conduirait à la longue à des changements tissulaires caractéristiques de la dédifférenciation cellulaire. Afin d'évaluer si ces concepts pouvaient être appliqués à la prédiction préopératoire du caractère réversible ou non des anomalies de cinétique segmentaire après revascularisation chez le malade coronarien, nous avons étudié plusieurs autres groupes de malades, dont certains avaient souffert d'un infarctus du myocarde au préalable et dont la plupart étaient porteurs d'une maladie coronaire atteignant deux ou trois des troncs principaux [9]. Sur la base de l'évolution de la fonction régionale dans le territoire revascularisé, nous avons séparé les malades étudiés en deux grandes catégories: ceux chez lesquels la fonction segmentaire s'était améliorée après revascularisation et qui étaient donc porteurs de myocarde hibernant; et ceux chez lesquels persistait la dysfonction malgré la revascularisation et dont on suppose qu'ils étaient porteurs d'une nécrose myocardique irréversible. Chez tous ces malades, nous nous sommes attachés à déterminer quels étaient les paramètres (parmi le flux sanguin myocardique, le captage de glucose exogène, l'ultrastructure au niveau des prélèvements biopsiques) permettant de différencier les malades dont la fonction allait s'améliorer après chirurgie de ceux chez lesquels la fonction resterait altérée malgré la revascularisation. Nous avons également cherché à identifier les facteurs déterminant l'amplitude et la vitesse de récupération fonctionnelle après revascularisation. Chez 24 malades étudiés par TEP avant l'intervention de pontage chirurgical et qui ont tous bénéficié d'une biopsie myocardique au moment de l'intervention chirurgicale, nous avons suivi de façon séquentielle, par échocardiographie, la récupération fonctionnelle, soit une semaine, deux mois et six mois après revascularisation [16].

\section{Flux et métabolisme à l'état basal}

Avant revascularisation, le myocarde dont la fonction devait s'améliorer après revascularisation était mieux perfusé $(83 \pm 23 \mathrm{ml} / \mathrm{min} / 100 \mathrm{~g})$ que le myocarde dont la dysfonction devait s'avérer permanente $(61 \pm$ $12 \mathrm{ml} / \mathrm{min} / 100 \mathrm{~g}, \mathrm{p}<0,01)$. De même, le caractère réversible de la dysfonction était associé à un captage glucidique plus élevé $(50 \pm 21 \mu \mathrm{mol} /$ $\mathrm{min} / 100 \mathrm{~g}$ ) que lorsque la dysfonction était irréversible $(30 \pm 13 \mathrm{\mu mol} /$ $\mathrm{min} / 100 \mathrm{~g}$ ).

\section{Corrélations ultrastructurales}

Sur le plan morphologique, le myocarde dont la dysfonction était réversible présentait significativement moins de fibrose $(24 \pm 13$ versus $49 \pm 20 \%, p<0,01)$ et plus de cardiomyocytes viables, y compris une plus grande proportion de cardiomyocytes altérés $(35 \pm 14$ versus $21 \pm 15 \%, \mathrm{p}<0,05)$ que le myocarde endommagé de façon irréversible. Sur un plan plus général, il existait une relation inverse significative entre la sévérité de la fibrose et le degré de perfusion résiduelle $(r=$ $-0,73, p<0,001)$, de même qu'entre le nombre de cardiomyocytes altérés et le captage de glucose exogène $(r=$ $0,63, \mathrm{p}<0,01$ ) (figure 5).

\section{Amplitude et vitesse de récupération fonctionnelle}

Afin de déterminer les facteurs responsables de l'amplitude totale de la récupération fonctionnelle six mois après revascularisation, les corrélations perfusionnelles, métaboliques et structurales de celle-ci ont été soumises à une analyse multivariée. De cette analyse, il ressort que l'amplitude de récupération est dépendante du degré de fibrose, des dimensions du ventricule gauche et de la perfusion résiduelle.

Enfin, chez les malades dont la dysfonction devait s'avérer réversible, nous avons étudié la cinétique de la récupération fonctionnelle après revascularisation [17]. Chez 18 malades dont le myocarde dysfonctionnel a récupéré après revascularisation (figure 6), la récupération fut progressive au cours des six mois de suivi postopératoire, environ $30 \%$ de l'amplitude totale de récupération survenant dès la première semaine suivant l'acte chirurgical, $30 \%$ supplémentaires entre deux et six mois après revascularisation, les $30 \%$ à $40 \%$ restant ne récupérant qu'entre le deuxième et le sixième mois suivant la revascularisation. Il faut cependant noter qu'il existait une grande variabilité interindividuelle en ce qui concerne la vitesse de récupération fonctionnelle après revascularisation, le seul facteur semblant déterminer cette dernière étant la sévérité des anomalies structurales des cardiomyocytes.

\section{Conclusions}

En conclusion, l'étude des corrélations entre perfusion, métabolisme et structure du myocarde hibernant chez l'homme nous a permis d'établir qu'il était caractérisé par: (1) une perfusion myocardique et un métabolisme oxydatif maintenus au repos; (2) une dépendance moindre du captage de glucose vis-à-vis de l'action de l'insuline; (3) une réserve de perfusion coronaire réduite; (4) des anomalies structurales des cardiomyocytes, probablement liées à un phénomène de dédifférenciation (5) une récupération lente et progressive de la fonction contractile 
après revascularisation, dont la vitesse est inversement proportionnelle à la sévérité des anomalies structurales présentées par les cardiomyocytes.

Les études futures devraient s'attacher à reproduire ce syndrome chez l'animal, ce qui permettrait d'étudier plus avant les mécanismes responsables du phénomène, aux trois niveaux cellulaire, moléculaire et génétique. Ces étapes nous paraissent un préalable essentiel à l'établissement de stratégies thérapeutiques dirigées et spécifiques

\section{TIRÉS À PART}

J.L. Vanoverschelde.

\section{Summary}

Chronic left ventricular ischemic dysfunction in coronary patients: insights into pathophysiological mechanisms

Previous studies have shown that chronic regional left ventricular dysfunction in patients with coronary artery disease does not always arise from irreversible tissue necrosis and, to some extent, can be reversed by restoration of blood flow. This condition, which is of ten referred to as «myocardial hibernation", has been proposed to result from the adaptation of the ischemic myocardium to a state of chronic hypoperfusion. Until now, elucidation of the exact mechanisms leading to hibernation has remained difficult, owing mainly to the lack of relevant animal models. Therefore, we performed the present studies in patients with chronic left ventricular ischemic dysfunction undergoing coronary revascularization, in whom we assessed how parameters of regional myocardial perfusion, metabolism and ultrastructure correlated with the reversibility of their dysfunction. We initially studied anginal patients without a previous infarction who had a chronic occlusion of a major coronary artery, and measured regional myocardial blood flow and metabolism with positron emission tomography. In these patients, despite coronary occlusion, myocardial perfusion and oxygen consumption in the dysfunctional segments were found to be normal. Yet, collateral flow reserve was markedly depressed, suggesting that repetitive episodes of ischemia with a persistent stunning effect was the most likely cause of dysfunction. As a second step, in the same patients, as well as in additional patients with a previous myocardial infarction, transmural myocardial biopsies were

obtained from the dysfunctional area at the time of bypass surgery. Analysis of these tissue samples demonstrated distinct morphological alterations in segments with improved function after revascularization, as compared to segments with persistent dysfunction, including less tissue fibrosis and more cardiomyocytes showing cellular swelling, loss of myofibrillar content and accumulation of glycogen. Also, samples from dysfunctional myocardium exhibited aspects of cellular dedifferentiation including fetal distribution of intracellular titin, absence of cardiotin and re-expression of the $\alpha$-smooth muscle cell actin. In a third study, we examined the timecourse of functional recovery of the dysfunctional segments after revascularization. The recovery of function was slowly progressive overtime, with as much as $30 \%$ of the total improvement taking place between the 2nd and the 6th month after revascularization. The rate of functional recovery was inversely related to the severity of ultrastructural abnormalities. Taken together, these clinical studies on the flow, metabolic and ultrastructural correlates of hibernating myocardium have provided new insights into the pathophysiology of reversible ischemic dysfunction. Repetitive ischemia and stunning appear to be the most likely cause of dysfunction, which is associated with structural abnormalities reminiscent of cell dedifferentiation. Further work should focus on designing appropriate animal models and on elucidating the molecular and genetic bases of this phenomenon. 